\title{
HRJ
}

V.3 n.14 (2022)

Recebido: 04/11/2021

Aceito: 01/12/2021

\section{Análise do clima de segurança em centros cirúrgicos de hospitais públicos do Distrito Federal}

\author{
Daniela Faria Lima ${ }^{1}$ \\ Verônica Santos de Melo ${ }^{2}$ \\ Mirce Meire Gonçalves de Sousa Wilk ${ }^{3}$ \\ Raquel Elisa de Almeida ${ }^{4}$ \\ Cleber Dos Santos Pinto ${ }^{5}$ \\ Ebert Mendonça ${ }^{6}$ \\ Fernanda Pereira De Oliveira ${ }^{7}$ \\ Francisca de Paula Carvalho Demes ${ }^{8}$ \\ Jacqueline Ramos de Andrade Antunes Gomes ${ }^{9}$ \\ Jardel Franco e Silva Anchieta ${ }^{10}$
}

\begin{abstract}
${ }^{1}$ Enfermeira. Residente do Programa de Residência de Enfermagem em Centro Cirúrgico da ESCS/FEPECS
${ }^{2}$ Enfermeira. Residente do Programa de Residência de Enfermagem em Centro Cirúrgico da ESCS/FEPECS

${ }^{3}$ Enfermeira. Tutora do Programa de Residência de Enfermagem em Centro Cirúrgico da ESCS/FEPECS

${ }^{4}$ Enfermeira. Tutora do Programa de Residência de Enfermagem em Centro Cirúrgico da ESCS/FEPECS

${ }^{5}$ Enfermeira. Preceptor do Programa de Residência de Enfermagem em Centro Cirúrgico da ESCS/FEPECS

${ }^{6}$ Enfermeira. Preceptor do Programa de Residência de Enfermagem em Centro Cirúrgico da ESCS/FEPECS

${ }^{7}$ Enfermeira. Preceptora do Programa de Residência de Enfermagem em Centro Cirúrgico da ESCS/FEPECS

${ }^{8}$ Enfermeira. Preceptora do Programa de Residência de Enfermagem em Centro Cirúrgico da ESCS/FEPECS

${ }^{9}$ Enfermeira. Coordenadora do Programa de Residência de Enfermagem em Centro Cirúrgico da ESCS/FEPECS

${ }^{10}$ Enfermeiro. Tutor do Programa de Residência de Enfermagem em Centro Cirúrgico da ESCS/FEPECS
\end{abstract}

\section{RESUMO}

Eventos adversos como as complicações cirúrgicas em pacientes hospitalizados estão entre as causas mais frequentes de morbimortalidade mundial. É necessário avaliar e monitorar a cultura de segurança do paciente no ambiente cirúrgico, para fornecer bases para o diagnóstico situacional e intervenções. O estudo teve como objetivo analisar o clima de segurança de centros cirúrgicos de Hospitais Públicos do Distrito Federal a partir da percepção dos profissionais da Enfermagem acerca da segurança do paciente. Foi utilizado como instrumento de coleta de dados o Questionário de Atitudes de Segurança/Versão Centro Cirúrgico. Trata-se de um estudo descritivo, seccional com abordagem quantitativa com profissionais da equipe de Enfermagem de dois Hospitais Públicos do Distrito Federal, realizado no período de julho a agosto de 2019. A avaliação do instrumento foi realizada por intermédio de escala tipo Likert e a análise de dados através do programa IBM Statistical Package for Social Sciences (SPSS), versão 21.0. Os resultados demonstraram que há necessidade de implementação de melhorias na cultura de segurança de forma sistemática, realizando intervenções de mudanças e análises periódicas para a continuidade das ações protetivas para a manutenção de uma cultura segura para o paciente.

Palavras chaves: Segurança do paciente; Cultura organizacional; Enfermagem de Centro Cirúrgico. 


\title{
Safety climate analysis in surgical centers of public hospitals in the Federal District
}

\begin{abstract}
Adverse events (AE) in hospitalized patients occupy the 14th place in the list of causes of worldwide morbidity and mortality, among these damages are surgical complications. When dealing with these aspects, it is necessary to assess and monitor the culture of patient safety in the surgical environment, opening opportunities to provide bases for situational diagnosis and interventions. The study aims to analyze the safety climate of surgical centers in Public Hospitals in the Federal District from the perspective of nursing professionals about patient safety. For this, the Safety Attitudes Questionnaire / Surgical Center Version (SAQ / VCC) was used as a data collection instrument. This is a descriptive, sectional study with a quantitative approach with a sample of 94 professionals from the Nursing team of two Public Hospitals in the Federal District, carried out from July to August 2019. The instrument was evaluated using a type scale Likert and data analysis using the IBM Statistical Package for Social Sciences (SPSS), version 21.0. The results showed that the general score (60.66) and the score for the six domains obtained values below the positive minimum for the safety culture, with the lowest scores in the dimensions of perception of professional performance (36.17) and perception of management ( 51.93). Concluding that there is a need to implement improvements in the safety culture in a systematic way, carrying out interventions for changes and periodic analyzes for the continuity of the protective actions to maintain a safe culture for the patient.
\end{abstract}

Key words: Patient safety; Organizational culture; Surgical Center Nursing.

\section{INTRODUÇÃO}

A segurança do paciente pode ser definida como uma conduta profissional em evitar, prevenir ou melhorar o acometimento de resultados adversos ao paciente no processo de atendimento médico hospitalar ${ }^{1}$. Eventos adversos (EA) em pacientes hospitalizados ocupam o $14^{\circ}$ lugar na lista de causas de morbimortalidade mundial. Um a cada dez pacientes sofrem algum tipo de dano durante uma internação, sendo que metade desses eventos são evitáveis. Entre esses danos estão os erros de medicação, infecções relacionadas à assistência à saúde, erros de diagnóstico e complicações cirúrgicas ${ }^{2}$.

Considerando essa problemática, a Organização Mundial da Saúde (OMS) criou, em 2004, a Aliança Mundial da Segurança do Paciente a fim de iniciar e coordenar o trabalho para desenvolvimento e disseminação de soluções para a segurança do paciente. Dentre os trabalhos chaves desta aliança, encontra-se a formulação dos chamados Desafios Globais para a Segurança do Paciente ${ }^{3}$. O primeiro, de 2005, enfatizou a prevenção de infecções associadas 
ao cuidado de saúde ${ }^{4}$; o segundo desafio global, de 2008, focou ações na promoção de cirurgias seguras $^{3}$; e o terceiro, de 2017 , destaca o uso seguro de medicamentos ${ }^{5}$.

Em 2013 o Ministério da Saúde instituiu o Programa Nacional de Segurança do Paciente (PNSP), com a finalidade de oferecer uma assistência segura, aprovando os protocolos básicos de segurança do paciente, profissionais de saúde e ambiente de assistência à saúde. Dentre os objetivos principais do programa, destaca-se a qualificação do cuidado em saúde a nível nacional. Para que haja uma relevância no âmbito da segurança do paciente é necessário priorizar a implementação de práticas de segurança e identificar estratégias unindo liderança e os profissionais atuantes no cuidado ${ }^{6}$.

O maior desafio encontrado nas equipes cirúrgicas é a relação interpessoal durante o trabalho em benefício ao paciente, pois há pouca orientação e estrutura para promoção de um trabalho multiprofissional que minimize riscos para o paciente. Não se pode atribuir uma única solução para melhorar a segurança cirúrgica, para poder alcançá-la é necessário investir em uma série de medidas que englobam toda a equipe atuante em benefício do paciente ${ }^{3}$. Dentre tais intervenções, destaca-se a necessidade de formação e análise da cultura e clima de segurança do paciente ${ }^{7}$.

Segundo a $\operatorname{RDC} \mathrm{n}^{\circ} .36 / 2013$, a cultura de segurança é definida como:

Conjunto de valores, atitudes, competências e comportamentos que determinam o comprometimento com a gestão da saúde e da segurança, substituindo a culpa e a punição pela oportunidade de aprender com as falhas e melhorar a atenção à saúde ${ }^{8}$.

A cultura de segurança engloba características operacionalizadas pela gestão de segurança, tais como: A cultura assumida pelos profissionais voltada pela a própria segurança, de terceiros e do paciente; A priorização da segurança acima de metas financeiras e operacionais; O encorajamento e a recompensa frente a identificação, notificação e resoluções dos problemas relacionados à segurança; Usufruir do aprendizado organizacional a partir da 
ocorrência de incidentes e a cultura que fornece recursos, estrutura e responsabilização para a manutenção efetiva da segurança ${ }^{8}$.

O clima e a cultura organizacional, apesar de serem utilizados como sinônimos, são conceitos diferentes, pois o clima organizacional faz referência ao conjunto de percepções e atitudes compartilhadas pelos indivíduos sobre o ambiente em que estão inseridos, enquanto o enfoque da cultura é na representatividade dos valores que permeiam o dia a dia na organização e das premissas básicas sobre o mundo ${ }^{9}$. Uma analogia que representa essa diferenciação é a imagem de um iceberg, a cultura compõe a parte submersa difícil de ser mensurada, porém poderosa e impactante para a segurança. $\mathrm{O}$ clima a parte externa, podendo assim ser melhor vista e mensurada ${ }^{10}$.

No ambiente cirúrgico são desenvolvidas atividades complexas com alto risco de EA, portanto necessita-se de profissionais habilitados não apenas tecnicamente, mas também nas relações de equipe, com um aprimoramento de uma comunicação eficaz que previna falhas nas relações e consequentemente no número de riscos de danos aos pacientes ${ }^{11}$. Portanto, é primordial a análise e monitoramento do clima de segurança para abrir oportunidades para fornecimento de bases para diagnóstico situacional e intervenções para melhorias como programas de educação continuada, implementação de protocolos assistenciais e monitoramento dos $\mathrm{EA}^{12}$.

Entretanto, para uma melhoria ou manutenção da cultura de segurança, é importante que seja realizada periodicamente a análise do clima de segurança por questionários validados sobre atitudes de segurança, para que haja conhecimento pelos profissionais sobre medidas de melhorias a serem adotadas para o diagnóstico da cultura de segurança e conscientização dos profissionais acerca do tema. Dentre os instrumentos mais utilizados no mundo para avaliação de cultura pode-se citar o Safety Attitudes Questionnaire (SAQ) Short Form, Sexton et al ${ }^{13}$ Questionário sobre atitudes de segurança desenvolvido por pesquisadores da Universidade do 
Texas com o objetivo principal de mensurar o clima de segurança percebido pelos profissionais da instituição avaliada. O instrumento Safety Attitudes Questionnaire/Operating Room (SAQ/OR) Kawano et al. ${ }^{14}$ é a versão modificada do SAQ adaptada para o ambiente cirúrgico mantendo suas propriedades psicométricas, com versão adaptada transculturalmente à realidade brasileira - Questionário de Atitudes de Segurança/Versão Centro Cirúrgico $(\mathrm{SAQ} / \mathrm{VCC})^{12}$

Considerando que com a análise do clima de segurança é possível diagnosticar e intervir em melhorias que envolvem a segurança do paciente, o presente estudo visa responder as seguintes questões: “Qual é a percepção dos profisssionais de enfermagem sob o clima de segurança em Centros Cirúrgicos?”

Diante do exposto, o presente trabalho tem como objetivo geral analisar o clima de segurança em centros cirúrgicos de hospitais públicos do Distrito Federal sob a percepção dos profissionais de Enfermagem (Enfermeiros, Técnicos de Enfermagem e Auxiliares de Enfermagem) acerca da segurança do paciente e como objetivo específico, identificar a concepção dos profissionais de Enfermagem sobre a postura da gerência, o sentimento de estresse, a condição de trabalho, a percepção do desempenho profissional e a comunicação no ambiente cirúrgico.

\section{METODOLOGIA}

Trata-se de um estudo descritivo, seccional com abordagem quantitativa, realizado nos dois centros cirúrgicos da Região de Saúde Sudoeste do Distrito Federal, pertencentes à Secretaria de Saúde. A coleta de dados foi iniciada no período de julho a agosto de 2019, com prévia autorização dos diretores dos hospitais e dos coordenadores dos centros cirúrgicos.

Foram convidados a participar do estudo 94 profissionais, sendo eles 16 Enfermeiros e 78 Técnicos de Enfermagem e Auxiliares de Enfermagem, servidores da Secretaria de Saúde 
do Distrito Federal com lotação nos centros cirúrgicos dos hospitais cenários do estudo. Foram incluídos profissionais da enfermagem servidores da Secretaria de Saúde do Distrito Federal, lotados nos centros cirúrgicos dos dois hospitais cenários do estudo, que aceitaram ao convite para participar da pesquisa e excluídos os profissionais da enfermagem que não aceitaram ao convite para participar da pesquisa ou os que estiveram afastados, de licença ou de férias no período do estudo.

Os dados foram coletados a partir da aplicação, em profissionais da enfermagem Enfermeiros, Técnicos de Enfermagem e Auxiliares de Enfermagem - do Questionário de Atitudes de Segurança/Versão Centro Cirúrgico (SAQ/VCC), uma versão traduzida e adaptada culturalmente do Safety Attitudes Questionnaire/Operating Room Version para o contexto brasileiro previamente autorizado pela autora. $\mathrm{O}$ instrumento tem o objetivo de mensurar o clima de segurança nos serviços de saúde a partir da percepção dos profissionais acerca da segurança do paciente ${ }^{12}$.

O questionário é estruturado em três partes. A primeira parte contém 15 itens referentes a qualidade da comunicação e entre os profissionais que atuam no ambiente cirúrgico, onde o participante da pesquisa deve responder sobre a sua relação com cada uma das categorias profissionais. A segunda parte é composta 40 itens que abrangem a segurança do paciente e um item questionando se o participante respondera a pesquisa anteriormente. A terceira parte é composta por informações demográficas, tais como gênero, raça/etnia, categoria profissional, tempo de atuação, turno de atuação, entre outros. Ao final do instrumento, há um espaço para exposição aberta de recomendações para melhoria da segurança do paciente em centro cirúrgico.

$\mathrm{O}$ questionário foi respondido por meio de escala tipo Likert. Na primeira parte do questionário os graus da escala tipo Likert apresentam-se da seguinte forma: "Muito Ruim”, "Ruim", “Adequada", "Boa", "Muito Boa”, "Não se Aplica”. Para a segunda parte, os graus 
da escala são representados por: "Discordo Totalmente", "Discordo Parcialmente”, "Neutro", "Concordo Parcialmente", "Concordo Totalmente" e "Não se Aplica". Cada item do questionário corresponde a um dos seis domínios do SAQ/CC, apresentados na Tabela 1 e descritos no Quadro 1.

Tabela 1 - Descrição dos domínios do SAQ/CC, São Paulo - 2014

\begin{tabular}{c|c}
\hline Domínios do SAQ/CC & Quantidade de ítens \\
\hline 1 Clima de segurança & 7 \\
2 Percepção da gerência & 5 \\
3 Percepção do estresse & 4 \\
4 Condição de trabalho & 6 \\
5 Comunicação no ambiente cirúrgico & 4 \\
6 Perpecção do desempenho profissional & 4 \\
\hline
\end{tabular}

Fonte: Instruções de aplicação e análise do SAQ/CC.

Quadro 1 - Descrição dos itens de cada domínio do SAQ/CC, São Paulo - 2014

\begin{tabular}{|c|c|}
\hline $\begin{array}{c}\text { Domínio do SAQ/ } \\
\text { CC }\end{array}$ & Descrição por ítem \\
\hline $\begin{array}{l}1 \text { Clima de } \\
\text { segurança }\end{array}$ & $\begin{array}{l}\text { 30.Todo o pessoal do Centro Cirúrgico assume responsabilidade pela } \\
\text { segurança do paciente } \\
\text { 35.Aqui existe adesão ampla às diretrizes clínicas e critérios baseados em } \\
\text { evidências relacionados com segurança do paciente } \\
\text { 38.As informações obtidas por meio dos relatórios de eventos adversos são } \\
\text { usadas para tornar a assistência do paciente mais segura neste Centro } \\
\text { Cirúrgico } \\
\text { 34.Assuntos importantes são bem comunicados nas mudanças de turno } \\
\text { 32.A segurança do paciente é, constantemente, reforçada como prioridade } \\
\text { aqui no Centro Cirúrgico } \\
\text { 28.O moral neste Centro Cirúrgico é alto } \\
\text { 29.A equipe médica deste Centro Cirúrgico faz um bom trabalho }\end{array}$ \\
\hline $\begin{array}{l}2 \text { Percepção da } \\
\text { gerência }\end{array}$ & $\begin{array}{l}\text { 6.A administração deste hospital apoia meus esforços diários } \\
\text { 5.A administração deste hospital está fazendo um bom trabalho } \\
\text { 7.Eu recebo retorno apropriado sobre o meu desempenho } \\
\text { 11.Este hospital é um bom lugar para se trabalhar } \\
\text { 14.Neste Centro Cirúrgico o número de profissionais é suficiente para atender } \\
\text { o número de pacientes }\end{array}$ \\
\hline $\begin{array}{l}3 \text { Percepção do } \\
\text { estresse }\end{array}$ & $\begin{array}{l}\text { 31.(R) Sinto-me cansado quando levanto pela manhã e tenho que enfrentar } \\
\text { outro dia de trabalho } \\
\text { 33.(R) Eu me sinto exausto com o meu trabalho } \\
\text { 37.(R) Sinto que estou trabalhando demais } \\
\text { 36.(R) Sinto me frustrado com o meu trabalho }\end{array}$ \\
\hline $\begin{array}{l}4 \text { Condição de } \\
\text { trabalho }\end{array}$ & $\begin{array}{l}\text { 17.A cultura de segurança neste Centro Cirúrgico torna fácil aprender com os } \\
\text { erros dos outros }\end{array}$ \\
\hline
\end{tabular}




\begin{tabular}{|c|c|}
\hline & $\begin{array}{l}\text { 23.Eu conheço os meios adequados para encaminhar questões relacionadas à } \\
\text { segurança do paciente neste Centro Cirúrgico } \\
\text { 22.Recebo informações adequadas e oportunas sobre ocorrências no hospital, } \\
\text { que podem afetar o meu trabalho } \\
\text { 18.O hospital lida de maneira construtiva com os profissionais problemáticos } \\
\text { 26.É fácil para os profissionais que atuam neste Centro Cirúrgico fazerem } \\
\text { perguntas quando existe algo que não entendem } \\
\text { 16.Sou encorajado por meus colegas a informar qualquer preocupação que eu } \\
\text { possa ter com a segurança do paciente }\end{array}$ \\
\hline $\begin{array}{l}5 \text { Comunicação } \\
\text { no ambiente } \\
\text { cirúrgico }\end{array}$ & $\begin{array}{l}\text { 19.Os equipamentos deste Centro Cirúrgico são adequados } \\
\text { 15.A tomada de decisão no Centro Cirúrgico utiliza informações dos } \\
\text { profissionais envolvidos } \\
\text { 9.A transmissão de informações entre os profissionais do Centro Cirúrgico } \\
\text { antes da realização de um procedimento cirúrgico é importante para a } \\
\text { segurança do paciente } \\
\text { 10.A transmissão de informações é comum no Centro Cirúrgico }\end{array}$ \\
\hline $\begin{array}{l}6 \text { Perpecção do } \\
\text { desempenho } \\
\text { profissional }\end{array}$ & $\begin{array}{l}\text { 24.(R) Sou menos eficiente no trabalho quando estou cansado } \\
\text { 21.(R) Quando a minha carga de trabalho se torna excessiva, meu } \\
\text { desempenho é prejudicado } \\
\text { 12.(R) O cansaço prejudica meu desempenho durante situações de } \\
\text { emergência } \\
\text { 25.(R) Eu tenho maior probabilidade de cometer erros em situações tensas ou } \\
\text { hostis }\end{array}$ \\
\hline $\begin{array}{c}*(\mathbf{N}) \text { Itens } \\
\text { reversos/negativos }\end{array}$ & \\
\hline
\end{tabular}

Fonte: Instruções de aplicação e análise do SAQ/CC.

Os profissionais foram convidados a participar do estudo com participação voluntária e a abordagem aconteceu durante seus respectivos turnos de trabalho. O questionário foi aplicado após o esclarecimento dos objetivos, riscos e benefícios do estudo, foi solicitada a assinatura voluntariamente do Termo de Consentimento Livre e Esclarecido.

Os dados foram analisados utilizando o programa IBM Statistical Package for Social Sciences (SPSS), versão 21.0. Para a análise da primeira etapa do SAQ/CC, cada alternativa recebeu uma pontuação, a saber: muito ruim $=0$ pontos; ruim $=25$ pontos; adequada $=50$ pontos; boa $=75$ pontos; muito boa $=100$ pontos. A alternativa "não se aplica" não foi considerada nas pontuações. O escore dado por cada participante foi a média da soma das pontuações dos itens respondidos.

Para análise da segunda etapa do questionário, cada alternativa recebeu a seguinte pontuação: discordo totalmente $=0$ pontos; discordo parcialmente $=25$ pontos; neutro $=50$ 
pontos; concordo parcialmente $=75$ pontos; concordo totalmente $=100$ pontos. A alternativa "não se aplica" não foi considerada nas pontuações. O cálculo do escore seguiu a seguinte ordem: primeiro os itens reversos/negativos, apresentados na Tabela 1, foram revertidos, ou seja, as respostas preenchidas com "discordo totalmente" passaram a ser "concordo totalmente", e assim analogamente em todos os itens. Na sequência os itens foram agrupados nos domínios, em seguida a pontuação de cada domínio foi obtida por meio do cálculo da média da soma das pontuações dos itens respondidos.

O escore da escala varia de 0 a 100, sendo que 0 representa a pior percepção do clima de segurança e 100 a melhor percepção. Valores maiores ou iguais a 75 são considerados uma percepção positiva da segurança do paciente.

A percepção da equipe de enfermagem de cada centro cirúrgico sobre o clima de segurança do setor foi calculada a partir de estatística descritiva, com frequência absoluta e relativa dos escores. Como medida de tendência central foi calculada a média entre os escores dados por cada participante e como medida de dispersão foi calculado o desvio padrão desses escores.

O profissional Perfusionista e Enfermeiro Anestesista são categorias que não fazem parte dos hospitais do estudo. De acordo com a autora, o Enfermeiro Anestesista, embora não exista no Brasil foi feita a opção de mantê-lo no instrumento pelos juízes devido à existência de cursos e a possibilidade de ser reconhecido futuramente.

O instrumento foi adaptado culturalmente para o emprego em todo o Brasil, dessa forma em algumas regiões ou instituições o enfermeiro se reconhece como "Enfermeiro de Centro Cirúrgico", "Enfermeiros Assistenciais da Recuperação Pós-Anestésica" ou "Enfermeiro Assistencial", assim, para análise dos dados essas categorias podem ser agrupadas como enfermeiro assistencial, se for o caso. 
Este projeto foi devidamente submetido ao Comitê de Ética em Pesquisa da Fundação de Ensino e Pesquisa em Ciências da Saúde - CEP/FEPECS sob o número CAAE: 13583619.9.0000.5553. A pesquisadora se compromete publicamente com as normas preconizadas pela Resolução CNS 466, de 12 de dezembro de 2012.

\section{RESULTADOS}

Neste estudo, dos 94 profissionais, 51 (54,26\%) responderam ao questionário, 39 $(41,49 \%)$ não aceitaram participar da pesquisa e 4 (4,25\%) eram servidores afastados. Dentre estes, $36(70,6 \%)$ se identificaram como Técnicos de Enfermagem, 11 (21,6\%) como Enfermeiros e 4 (7,8\%) não se identificaram.

No que diz respeito às variáveis sociodemográficas, o regime de trabalho constatou que $20(39,3 \%)$ dos participantes responderam atuar em regime integral, já no que se concerne ao turno habitual de trabalho o item "turnos variáveis" foi o mais representativo com 22 $(43,1 \%)$ dos participantes. Tratando-se da variável "tempo de experiência na especialidade", a média foi de aproximadamente 15 anos e o tempo de trabalho dos profissionais no hospital do estudo foi em média 14 anos. Os profissionais eram em sua maioria do sexo feminino 41 (80,39\%), do grupo étnico pardo 22 (43,15\%), com média de idade de 42 anos.

Tabela 2 - Características sociodemográficas dos profissionais de Enfemagem de Centros Cirúrgicos de

Hospitais Públicos, Brasília - 2019

\begin{tabular}{|c|c|}
\hline Características sociodemográfica & n $(\%)$ \\
\hline \multicolumn{2}{|c|}{ Cargo $(n=51)$} \\
\hline Enfermeiro & $11(21,6)$ \\
\hline Técnico de Enfermagem & $36(70,6)$ \\
\hline Não respondido & $4(7,8)$ \\
\hline \multicolumn{2}{|c|}{ Regime de trabalho } \\
\hline Integral & $20(39,3)$ \\
\hline Parcial & $8(15,7)$ \\
\hline
\end{tabular}




\begin{tabular}{|c|c|}
\hline $\begin{array}{c}\text { Contratado } \\
\text { Outros } \\
\text { Não respondido }\end{array}$ & $\begin{array}{c}1(2) \\
14(27,4) \\
8(15,7)\end{array}$ \\
\hline \multicolumn{2}{|c|}{ Turno habitual } \\
\hline Integral & $11(21,6)$ \\
\hline Parcial & $4(7,8)$ \\
\hline Turnos variáveis & $22(43,1)$ \\
\hline Noturno & $7(13,7)$ \\
\hline Não respondido & $7(13,7)$ \\
\hline \multicolumn{2}{|c|}{ Grupo étnico } \\
\hline Branco & $18(35,3)$ \\
\hline Pardo & $22(43,15)$ \\
\hline Negro & $7(13,7)$ \\
\hline Amarelo & $1(2)$ \\
\hline Não respondido & $3(5,9)$ \\
\hline \multicolumn{2}{|c|}{ Cidadania } \\
\hline Brasileira & $19(37,3)$ \\
\hline Não respondido & $32(62,7)$ \\
\hline \multicolumn{2}{|c|}{ Sex $)$} \\
\hline Feminino & $41(80,4)$ \\
\hline Masculino & $9(17,6)$ \\
\hline Não respondido & $1(2)$ \\
\hline \multicolumn{2}{|c|}{ Idade atual } \\
\hline 20-29 anos & $1(2)$ \\
\hline 30-39 anos & $18(35,3)$ \\
\hline 40-49 anos & $17(33,3)$ \\
\hline 50-59 anos & $7(13,7)$ \\
\hline Não respondido & $8(15,7)$ \\
\hline \multicolumn{2}{|c|}{ Tempo de experiência na especialidade } \\
\hline $1-9$ anos & $12(23,5)$ \\
\hline 10-19 anos & $22(43,1)$ \\
\hline $20-29$ anos & $10(19,6)$ \\
\hline $30-39$ anos & $3(5,9)$ \\
\hline Não respondido & $4(7,8)$ \\
\hline \multicolumn{2}{|c|}{ Tempo de trabalho no hospital } \\
\hline $0-9$ meses & $1(2)$ \\
\hline $1-9$ anos & $16(31,4)$ \\
\hline $10-19$ anos & $19(37,2)$ \\
\hline $20-29$ anos & $5(9,8)$ \\
\hline 30-39 anos & $3(5,9)$ \\
\hline 40-49 anos & $2(3,9)$ \\
\hline Não respondido & $5(9,8)$ \\
\hline
\end{tabular}

Fonte: Dados da pesquisa, 2019. 
O gráfico 1 apresenta o escore da percepção da equipe de Enfermagem acerca da qualidade da comunicação e da colaboração vivida em relação aos profissionais do Centro Cirúrgico, trazendo a melhor percepção para a categoria Instrumentador e Circulante de sala $(84,18)$ e a pior percepção encontrada para Residente de Cirurgia e Interno $(63,7)$.

Gráfico 1- Escore da percepção da equipe de Enfermagem acerca da qualidade da comunicação e da colaboração vivida em relação aos profissionais do Centro Cirúrgico, Brasília - 2019.

Escore - Qualidade da comunicação e colaboração

Instrumentador e Circulante de Sala $(84,18)$

Enfermeiro Chefe de Centro Cirúrgico $(81,58)$

Enfermeiro Assistencial $(80,78)$

Auxiliar de Anestesiologista $(80,47)$

Equipe de Apoio $(79,05)$

Residente de Anestesia e Interno (77,08)

Anestesiologista/Assistente $(72,87)$

Equipe de avaliação pré-operatória/pré-anestésica $(67,95)$

Cirurgião/Assistente $(64,29)$

Residente de Cirurgia e Interno $(63,78)$

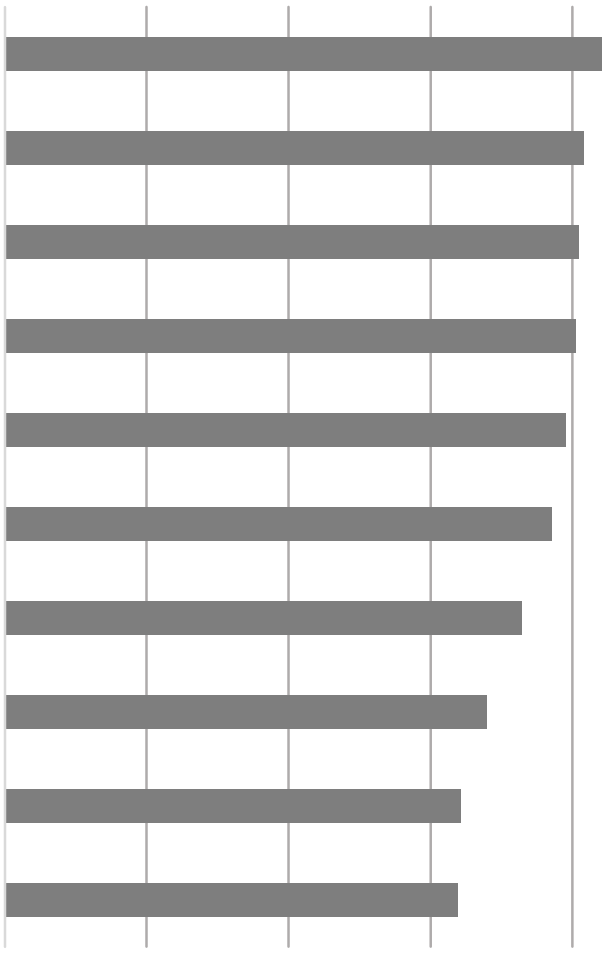

0

20

40

60

80

100

Fonte: Dados da pesquisa, 2019.

A tabela 3 apresenta os escores dos seis domínios e o escore geral $(60,66)$ que compõem a análise da segurança do paciente no Centro Cirúrgico avaliada por todos os profissionais. O maior escore encontrado foi no domínio 5: Comunicação $(66,43)$ e o menor no domínio 6: Percepção do desemprenho profissional $(36,17)$. 
Tabela 3 - Média do escore geral e por domínio com desvio padrão (DP) do SAQ/CC na percepção da equipe de Enfermagem, Brasília - 2019.

\begin{tabular}{c|c|c}
\hline Domínio & Escore & DP \\
\hline 1 - Clima de segurança & 65,17 & 6,09 \\
2 - Percepção da gerência & 51,93 & 13,90 \\
3 - Percepção do estresse & 61,94 & 3,45 \\
4 - Condições de trabalho & 59,38 & 15,15 \\
5 - Comunicação & 66,43 & 21,09 \\
6- Percepção do desemprenho profissional & 36,17 & 5,56 \\
Total & 60,66 & 11,35 \\
\hline
\end{tabular}

Fonte: Dados da pesquisa, 2019.

A tabela 4 apresenta a média da percepção por domínio de cada categoria profissional (Enfermeiros ou Técnicos e Auxiliares de Enfermagem). Na elaboração da tabela foram inseridos os profissionais que se identificaram como Enfermeiros (11), Técnicos e Auxiliares de Enfermagem (36) e desconsiderados os que não se identificaram (4). Considerando o critério para percepção positiva a obtenção do escore $\geq 75$, nenhuma das categorias avaliou como positivo os domínios.

Tabela 4 - Média dos escores por domínio do SAQ/CC na percepção das categorias Enfemeiros e Técnicos e Auxiliares de Enfermagem com desvio padrão (DP), Brasília - 2019.

\begin{tabular}{c|c|c|c|c}
\hline Domínios & Enfermeiros & DP & $\begin{array}{c}\text { Técnicos e } \\
\text { Auxiliares de } \\
\text { Enfermagem }\end{array}$ & DP \\
\hline 1 - Clima de segurança & 55,62 & 11,03 & 67,46 & 5,1 \\
2- Percepção da gerência & 41,45 & 20,3 & 54,39 & 14,7 \\
3- Percepção do estresse & 64,14 & 6,17 & 62,77 & 2,01 \\
4 - Condições de trabalho & 47,99 & 18,76 & 61,65 & 14,59 \\
5 - Comunicação & 59,54 & 27,91 & 68,54 & 20,45 \\
6- Percepção do desemprenho profissional & 33,52 & 11,79 & 37,37 & 7,12 \\
\hline
\end{tabular}
Fonte: Dados da pesquisa, 2019.

$\mathrm{O}$ instrumento de análise $(\mathrm{SAQ} / \mathrm{CC})$ permitia que os participantes tecessem recomendações para aprimorar a segurança do paciente do paciente no Centro Cirúrgico. Os 
comentários englobavam em sua maior parte a comunicação entre equipe, capacitação/treinamento/educação continuada, avaliação pré-operatória (cirúrgica, anestésica e no centro cirúrgico), insumos, manutenção, reformas e gestão, e algumas dessas informações estão descritas abaixo:

P1: "Teria que ter mais comunicação e respeito entre todas as áreas".

P2: “Atualização profissional de forma individual e em grupo”.

P3: "Melhorar o atendimento no pré-operatório”.

P4: "Melhoria de material/manutenção".

P5: "Melhoria de estrutura física".

P6: "Melhor atuação da CCIH + Comissão de segurança do paciente”.

\section{DISCUSSÃO}

Os resultados deste estudo permitem o conhecimento das características sociodemográficas dos profissionais que atuam nestes $\mathrm{CC}$, dentre elas, destacam-se o expressivo número de técnicos em Enfermagem (70,6\%) atuantes no setor que acompanha um estudo da FIOCRUZ, que traça o perfil da Enfermagem no Brasil. Esta pesquisa evidencia que a Enfermagem no país é atualmente composta por um quadro de $80 \%$ de técnicos e auxiliares e $20 \%$ de enfermeiros, sendo uma equipe predominantemente feminina, composta por $84,6 \%$ de mulheres, assim como no presente estudo ${ }^{15}$.

A média da faixa etária do estudo encontrada foi de 42 anos de idade, assemelhandose a um outro estudo brasileiro que aponta a enfermagem como uma profissão em pleno rejuvenescimento, sendo composta por $40 \%$ dos profissionais com idade entre 36-50 anos. Profissionais nessa faixa etária, são caracterizados pelo estudo como a fase de 'Maturidade profissional', estando em pleno desenvolvimento de suas capacidades (cognitivas, técnicas e 
práticas de enfermagem), assim como, inseridos no mercado de trabalho com escolhas lógicas e racionais, passando a ter domínio de suas habilidades e destrezas cognitivas ${ }^{16}$.

$\mathrm{Na}$ análise do grupo étnico, foi predominante na pesquisa o grupo de pardos $(43,15 \%)$ e brancos (35,3\%), em contrapartida, o estudo do Perfil da Enfermagem no Brasil aponta maior predominância entre pardos $(41,5 \%)$ e pretos $(11,5 \%)$, juntos apresentando um percentual de $53 \%$, sendo os grupos mais expressivos e dominantes no quesito raça/cor ${ }^{16}$.

A percepção da equipe de enfermagem quanto à qualidade de comunicação e colaboração vivida com os profissionais do Centro Cirúrgico foi positiva em relação aos profissionais Instrumentador e Circulante de sala, Enfermeiro Chefe de Centro Cirúrgico, Enfermeiro assistencial, Auxiliar de Anestesiologista, Equipe de apoio, Residente de Anestesia e Interno com escores $\geq 75$. No entanto, a visão da equipe de enfermagem em relação ao Anestesiologista/Anestesiologista Assistente, Equipe de avaliação préoperatória/pré-anestésica, Cirurgião/Assistente, Residente de cirurgia e Interno não foram positivas, o que caracteriza fragilidades no processo de comunicação e colaboração.

A comunicação e colaboração entre equipes multiprofissionais é um desafio, pois o cuidado em saúde exige a atuação conjunta de várias categorias e especialidades, na qual a hierarquia resultante de uma cultura profissional e institucional leve a alguns profissionais apresentarem dificuldade em se expressarem abertamente com outras classes. Essas barreiras são facilitadoras de erros, portanto é necessário que haja valorização da pessoa e do respeito mútuo, permitindo uma comunicação confortável, distanciando relações hierárquicas e de poder. É importante salientar que a baixa avaliação entre profissionais, pode gerar implicações para a segurança do paciente, pois podem acarretar em fontes potenciais para erros, quebras na continuidade dos serviços prestados, tratamento e procedimentos inadequados ou pouco eficazes, com consequências imediatas e potencialmente negativas ao paciente. $O$ trabalho em equipe com entreajuda, abre portas para a colaboração, com oposição 
a competitividade ou passividade, esse apoio é fundamental para antecipar e suprir as necessidades da equipe ${ }^{17}$.

Considerando o escore geral $(60,66)$ apresentou valor inferior a 75 , não alcançando o valor mínimo para uma percepção positiva para cultura de segurança, assim como estudos brasileiros, demonstrando a necessidade de aprimoramento de aspectos relevantes para cultura de segurança do paciente ${ }^{11,18}$.

Para que aconteça uma melhoria ou sustentação da cultura de segurança nos serviços de saúde é necessário que ocorram mudanças multifatoriais que envolvam o desenvolvimento da liderança, para que os líderes estejam envolvidos nos processos de iniciação e transformação da cultura. Estas estruturações de liderança devem ser instituídas com a função de sensibilizar, responsabilizar, habilitar e para que haja ações favoráveis à segurança do paciente. A realização periódica de avaliação da cultura de segurança, lançando mão de instrumentos como o utilizado nesse estudo que envolvem dimensões importantes para uma estrutura de cultura de segurança, além de implementar, informar os resultados aos profissionais e gestores para a realização de melhorias necessárias. É necessário identificar erros e riscos com enfoque na redução de danos e para a melhoria do desempenho profissional e redução dos erros preveníveis, um enfoque maior na promoção do trabalho em equipe de forma sistemática. Além disto, o serviço de saúde deve contar com um sistema de vigilância eficaz, com acompanhamento para identificação de possíveis problemas para a segurança, reforçando a importância de acompanhamento de bons indicadores ${ }^{7}$.

Os seis domínios analisados no estudo obtiveram escore negativo, assim como um estudo semelhante evidenciado a necessidade de aprimoramento de cada domínio que envolve a segurança de paciente no Centro Cirúrgico ${ }^{19}$.

Em relação ao domínio "Clima de segurança”, os resultados trazem uma alerta para questões como moral, trabalho médico, responsabilidade da equipe pela segurança do 
paciente, prioridade na segurança do paciente, comunicação entre a equipe e notificações de EA, pois uma cultura que possui atributos de alto nível, deve ser empenhada com profissionais de saúde que colocam em prática a implementação de sistemas sólidos de gerenciamento de segurança, onde todos os profissionais assumem responsabilidade pela própria segurança, a dos colegas de trabalho, a dos pacientes e a dos visitantes. Além de estimular e recompensar a identificação, comunicação e resolução de questões de segurança que estabelece uma base de comunicação franca e confiável, podendo ser adotada para reconhecer a situação organizacional formando uma equipe sem receios de punição por divulgar falhas ${ }^{20,21}$.

A percepção da gerência foi avaliada com questionamentos que envolvem a administração do hospital, apoio e retorno aos esforços diários, bom ambiente de trabalho e número suficiente de recursos humanos para atendimento aos pacientes. Autores citam que profissionais assistenciais podem se sentir distantes das tomadas de decisões, da elaboração de estratégias, reduzindo sua autonomia e gerando insatisfações às ações da gerência ${ }^{22,23}$.

O apoio da gestão é um importante pilar para a segurança do paciente, entretanto pode ser distanciada da assistência como foi evidenciado em um estudo que indica que há falhas na comunicação de protocolos de segurança, políticas e expectativas, já que o distanciamento da gestão com a assistência dificulta a vivência de oportunidades de identificar riscos que são vividos no cotidiano da unidade. Toda esta barreira encontrada, explana a necessidade de uma maior proatividade para melhorias de coordenação entre unidade e gestão ${ }^{24}$. Além disso, a sobrecarga de trabalho também é um fator que causa insatisfação à gestão hospitalar, comprovada em um estudo, a percepção da gerência de forma negativa, pois apesar da Enfermagem ter em mãos uma ferramenta para gestão de recursos humanos com respaldo na legislação do que rege a profissão, a maior parte das instituições de saúde não conseguem 
alcançar o quantitativo adequado, culminando em déficit de recursos humanos e riscos para a segurança do paciente ${ }^{18,25}$.

A Percepção do estresse entre os participantes evidencia o entendimento de fatores que comprometem a cultura de segurança. Fatores como cansaço, exaustão, frustação e excesso de trabalho foram avaliados nesta dimensão, que pode ser melhor entendida por um estudo semelhante que traz fatores referentes aos recursos humanos que influenciam no desenrolar do cuidado seguro, fatores estes, que estão relacionados ao reconhecimento do estresse pela fadiga por carga horária excessiva, escassez de recursos humanos, dificuldade nas relações interpessoais, erros de julgamento, problemas com materiais e equipamentos que precisam de soluções, culminando em barreiras para as ações protetivas ao cuidado seguro do paciente. Sabe-se que diversos profissionais estão predispostos a cometer erros justificados por estes fatores, e tendem a trabalhar com descuidos, cometendo erros ${ }^{11,26,27}$.

$\mathrm{Na}$ avaliação das condições de trabalho, que também não obtiveram resultado positivo, foram abordados aspectos que envolvem o encorajamento na disseminação de informação que envolvem o paciente, o aprendizado com os erros, a forma que o hospital lida com os profissionais problemáticos, a divulgação de informações adequadas de ocorrências no hospital, o conhecimento de meios adequados para encaminhar questões relacionadas à segurança do paciente e sobre a abertura para esclarecimento de dúvidas. Um estudo brasileiro mostra que o erro foi abordado como um fator menos relevantes para a segurança do paciente pelos profissionais, entretanto, o erro ainda é muito associado à culpa, pois o cenário atual costuma ser punitivo e apontam os erros como frutos de descuidos. Portanto, a compreensão inadequada sobre erros, pode culminar em sentimentos negativos como vergonha, medo e culpa, o que gera uma quebra nos processos de discussão e disseminação de informações, tomando uma direção negativa à segurança do paciente ${ }^{28,29}$. 
A comunicação foi avaliada de forma que desfavorece a segurança do paciente, o que pode ser observado em uma pesquisa realizada em Santa Catarina, que traz a comunicação de forma negativa, com pouca divulgação de informações entre a equipe. A comunicação é um instrumento essencial em um trabalho em equipe, garantindo a continuidade do cuidado e possibilitando o conhecimento de toda equipe sobre o que está sendo realizado com o paciente. Para que aconteça uma comunicação bem sucessida, é necessário que haja recursos que contribuam para que a mesma aconteça como instituir uma cultura de passagem de plantão e de casos, o incentivo e treinamento para bom uso de sistemas de informações, para que haja fortalecimento e encorajamento dos profissionais para melhoria do cuidado através de uma comunicação eficaz ${ }^{30,31}$.

O desempenho profissional foi avaliado no estudo englobando fatores influenciadores como cansaço, carga horária excessiva e situações tensas e hostis. Como nos outros domínios, este também foi avaliado de forma não positiva pelos profissionais, assim como um estudo que aplicou o mesmo instrumento, os profissionais não consideram de maneira efetiva que situações tensas e hostis causam propensões para erros, influenciando negativamente para a segurança do paciente. Além desses fatores, o cansaço oriundo de sobrecarga de trabalho, excesso de tarefas, necessidade de cobrir escalas, aumentam a fadiga do profissional dificultando um cuidado protetivo ${ }^{11,26}$.

Os registros de melhorias apontados abertamente pelos profissionais na terceira etapa do SAQ/CC apresentaram comentários que estão fortemente relacionados à comunicação e interação de respeito entre a equipe 'Teria que ter mais comunicação e respeito entre todas as áreas', a organização do trabalho 'Melhorar o atendimento no pré-operatório', a harmonização entre o trabalho multidisciplinar 'Atualização profissional de forma individual e em grupo', aos materiais e estrutura física 'Melhoria de material/manutenção', 'Melhoria de estrutura física' e a gestão hospitalar que atua diretamente na segurança do paciente 
'Melhor atuação da CCIH + Comissão de segurança do paciente'. Estas recomendações estão direcionadas a melhorias de condições e das relações de trabalho, assim como em um estudo que revela que os recursos disponíveis como equipamentos e materiais, relações bem estabelecidas e um ambiente com um bom trabalho em equipe estão relacionadas à uma satisfação no trabalho, ao prazer e ao reconhecimento do trabalho executado ${ }^{32}$.

\section{CONCLUSÃO}

A utilização do SAQ/CC possibilitou um diagnóstico situacional do clima de segurança a partir das percepções dos profissionais do estudo, demonstrando fragilidades em todos as dimensões analisadas.

O estudo possibilitou a identificação de um escore geral negativo a partir da análise dos profissionais da Enfermagem acerca da segurança do paciente, demonstrando a necessidade de implementação de melhorias na cultura de segurança de forma ampla e integral, não apenas realizando intervenções de mudanças, mas também de uma análise periódica para a continuidade das ações protetivas para a manutenção de uma cultura segura para o paciente.

O escore negativo dos seis domínios apontou fragilidades na percepção que a equipe de enfermagem tem sobre a postura da gerência, sentimento de estresse, as condições de trabalho, a percepção do desempenho profissional e a comunicação no ambiente cirúrgico, demonstrando que os desafios que permeiam a temática da segurança do paciente são de grandes dimensões e contínuos, pois envolvem as fragilidades da condição humana, a cultura pessoal e local, o ambiente que o profissional está inserido, as lideranças, gestões, estruturas físicas, materiais de trabalho, recursos humanos e diversos outros fatores influenciadores.

O uso de um instrumento validado pode contribuir para a equipe como um todo no sentido de obter um diagnóstico preciso sobre o ambiente de segurança nos grupos de 
trabalho, fator que é fundamental para qualificar a assistência perioperatória ao paciente de forma segurança.

\section{REFERÊNCIAS}

1. Vicent C. Segurança do paciente: orientações para evitar eventos adversos. São Caetano do Sul: Yendis; 2009.

2. Organização Mundial da Saúde. Guia curricular de segurança do paciente da Organização Mundial da Saúde: edição multiprofissional. Rio de Janeiro: Autografia; 2016.

3. Organização Mundial da Saúde. Cirurgias Seguras Salvam Vidas. Segundo desafio global para segurança do paciente 2009.

4. World Health Organization. World Alliance for Patient Safety. First Global Patient Safety Challenge: Clean Care is Safer Care. Geneva: World Health Organization; 2006.

5. World Health Organization. Global Patient Safety Challenge [Internet]. Geneva: Medication Without Harm; 2017 [Acesso em 18 Dez 2019]. Disponível em <http://apps.who.int/iris/bitstream/handle/10665/255263/WHO-HIS-SDS-2017.6 eng.pdf;jsessionid=CD809AD120A1580718D0603566BE5BB6? sequence $=1$.

6. Brasil. Ministério da Saúde. Portaria $n^{\circ} 529$, de $1^{\circ}$ de abril de 2013. Institui o Programa Nacional de Segurança do Paciente (PNSP). Brasília: Diário Oficial da União, 2013.

7. Brasil. Agência Nacional de Vigilância Sanitária. Implantação do Núcleo de Segurança do Paciente em Serviços de Saúde. Brasília: Anvisa; 2014.

8. Brasil. Ministério da Saúde. Resolução de diretoria colegiada - Rdc no 36, de 25 de julho de 2013. Institui ações para a segurança do paciente em serviços de saúde e dá outras providências. Brasília: Diário Oficial da União; 2013. 
9. Colla JB, Bracken AC, Kinney LM, Weeks WB. Measuring patient safety climate: a review of surveys. BMJ Quality \& Safety [internet], 2005 [Acesso em 11 Jan. 2020], 14(5): 364-366. Disponível em: https://www.ncbi.nlm.nih.gov/pubmed/16195571.

10. Schein EH. Cultura organizacional e liderança. São Paulo: Atlas; 2009.

11. Da Correggio TC, Amante LN, Barbosa SFF. Avaliação da cultura de segurança do paciente em Centro Cirúrgico. Rev. SOBECC. 2014; 19(2): 67-73.

12. Lourenção DCA, Tronchin DMR. Segurança do paciente no ambiente cirúrgico: tradução e adaptação cultural de instrumento validado. Acta Paul Enferm. 29 (1). [Internet]. JanFeb 2016. [Acesso em 21 jun. 2021]. https://doi.org/10.1590/1982-0194201600002

13. Sexton JB, Helmreich RL, Neilands TB, Rowan K, Vella K, Boyden J, et al. The Safety Attitudes Questionnaire: psychometric properties, benchmarking data, and emerging research. BMC health services research [Internet]. 2006 [Acesso e 22 Jan. 2020]; 6(1): 44. Disponível em: https://www.ncbi.nlm.nih.gov/pmc/articles/PMC1481614/.

14. Kawano T, Taniwaki M, Ogata K, Sakamoto M, Yokoyama M. Improvement of teamwork and safety climate following implementation of the WHO surgical safety checklist at a university hospital in Japan. Journal of anesthesia [Internet]. 2014 [Acesso em 22 maio 2021]; 28(3): 467-470. Disponível em: https://www.ncbi.nlm.nih.gov/pubmed/24170220.

15. Fiocruz. Pesquisa inédita traça perfil da enfermagem no Brasil. Agência Fiocruz de Notícias [Internet]. 2015 [Acesso em 22 out. 2018]. Disponível em: https://portal.fiocruz.br/ptbr/content/pesquisa-inedita-traca-perfil-da-enfermagem-no-brasil.

16. Machado MH, Filho WA, Lacerda WF, Oliveira E, Lemos W, Wermelinger M, et al. Características gerais da enfermagem: o perfil sócio demográfico. Enfermagem em Foco. [Internet] 2016 [Acesso em 22 mai. 2021; 7(1): 9-14. DOI: https://doi.org/10.21675/2357$\underline{707 X .2016 . v 7 . n E S P .686}$ 
17. Santos MC, Grilo A, Andrade G, Guimarães T, Gomes A. Comunicação em saúde e a segurança do doente: problemas e desafios. Revista portuguesa de saúde pública. [Internet] 2010; [Acesso em 22 maio 2021]; 1(10): 47-57. Disponivel em: https://repositorio.ipl.pt/bitstream/10400.21/3120/1/Comunica\%c3\%a7\%c3\%a3o\%20em\%20 sa\%c3\%bade $\% 20 \mathrm{e} \% 20 \mathrm{a} \% 20$ seguran $\%$ c3\%a7a\%20do\%20doente.pdf.

18. Marinho MM, Radünz V, Barbosa SFF. Avaliação da cultura de segurança pelas equipes de enfermagem de unidades cirúrgicas. Texto \& Contexto Enfermagem. [Internet] 2014; [Acesso em 22 maio 2021]; 23(3): 581-590. Doi: http://dx.doi.org/10.1590/0104$\underline{07072014002640012}$

19. Matiello RDC, Lima EFA, Coelho MCR, Oliveira ERA, Leite FMC, Primo CC. A cultura de segurança do paciente na perspectiva do enfermeiro. Cogitare enferm. [periódico da Internet] 2016 [acesso em 22 mai. 2021] ; 21(1): 1-9. Disponivel em: https://revistas.ufpr.br/cogitare/article/view/45408/pdf

20. National Patient Safety Foundation. Renal Physicians Association. Forum and End Stage Renal Disease Networks. National ESRD Patient Safety Initiative: Phase II Report. Chicago: National Patient Safety Foundation; 2001.

21. Health and Safety Executive. Human Factors Briefing Note. n.7. Safety Culture; [Internet] 2018 [Cited 2021 mai 22]. Available from http://www.hse.gov.uk/humanfactors/topics/ 07culture.pdf.

22. Luiz RB, Simões ALA, Barichello E, Barbosa MH. Fatores associados ao clima de segurança do paciente em um hospital de ensino. Revista Latino-Americana de Enfermagem. [Internet] 2015[Acesso em 22 maio 2021]; 23(5): 880-887. DOI: 10.1590/0104-1169.0059.2627 
23. Relihan E, Glynn S, Daly D, Silke B, Ryder S. Measuring and benchmarking safety culture: application of the safety attitudes questionnaire to an acute medical admissions unit. Ir J Med Sci. [Internet] 2009[cited em 22 mai 2021]; 178(4):433-439. doi:10.1007/s11845-009-0352-2

24. Mello JF, Barbosa SFF. Cultura de segurança do paciente em terapia intensiva: recomendações da enfermagem. Texto \& Contexto-Enfermagem. [Internet] 2013[Acesso em 22 maio 2021]; 22(4): 1124-1133. https://doi.org/10.1590/S0104-07072013000400031

25. Quinto Neto A. Segurança dos pacientes, profissionais e organizações: um novo padrão de assistência à saúde. RAS. [Internet] 2006; [Acesso em 22 maio 2021]; 8(33): 1538.Disponivel em : http://www.nascecme.com.br/artigos/RAS33_seguranca.pdf

26. Silva BM, Lima FRF, Farias FSAB, Campos ACS. Jornada de trabalho: fator que interfere na qualidade da assistência de enfermagem. Texto contexto - enferm. [Internet]. 2006 [Acesso em 22 maio 2021]; 15(3): 442-448. Disponível em: http://www.scielo.br/ scielo.php?script=sci_arttext\&pid=S0104-07072006000300008\&lng=en.

27. Misiak M. Cultura de segurança do paciente na perspectiva da equipe de enfermagem de uma instituição cardiovascular. Dissertação (mestrado) - Universidade Federal de Santa Catarina, Centro de Ciências da Saúde. Programa de Pós-Graduação em Enfermagem, Florianópolis, 2013.

28. Fidelis RE. Cultura de segurança: perspectiva da equipe de enfermagem em unidade de emergência adulto. Dissertação [Mestrado] - Universidade Federal de Santa Catarina, Florianópolis, 2011.

29. Duarte SCM, Stipp MAC, Silva MM, Oliveira FT. Adverse events and safety in nursing care. Rev. Bras. Enferm. [Internet]. 2015 [Acesso em 22 maio 2021]; 68(1): 144-154. Disponível em: http://www.scielo.br/scielo.php?script=sci_arttext\&pid=S0034$71672015000100144 \& \operatorname{lng}=\mathrm{en}$. 
30. Sartor GD, Da Silva BF, Masiero AV. Segurança do paciente em hospitais de grande porte: panorama e desafios. Cogitare Enfermagem, [Internet] 2016, 21(5).

31. Aguiar LB. Cultura e clima de segurança do paciente entre os profissionais de enfermagem de um hospital privado do Distrito Federal. Dissertação [Mestrado] - Universidade de Brasília, Faculdade Ceilândia, Brasília, 2019.

32. Baratto MAM. Cultura de segurança do paciente: percepções e atitudes dos trabalhadores nas instituições hospitalares de Santa Maria. Dissertação [Mestrado] - Universidade Federal de Santa Maria, Centro de Ciências da Saúde, Santa Maria, 2015. 